\title{
The DEuterated SCintillator Array for Neutron Tagging
}

\section{A neutron tagging array for TRIUMF-ISAC}

J. Wong ${ }^{1, a}$, V. Bildstein ${ }^{1}$, P.E. Garrett ${ }^{1}$, D. Bandyopadhyay ${ }^{1}$, J. Bangay ${ }^{1}$, L. Bianco ${ }^{1}$, G. Demand ${ }^{1}$, G. Deng ${ }^{1}$, A. Finlay ${ }^{1}$, B. Hadinia ${ }^{1}$, K.G. Leach ${ }^{1}$, A. Liblong ${ }^{1}$, C.E. Svensson ${ }^{1}$, C. Sumithrarachchi ${ }^{1}$, G.C. Ball ${ }^{2}$, R. Churchman ${ }^{2}$, A. Garnsworthy ${ }^{2}$, G. Hackman ${ }^{2}$, C.J. Pearson ${ }^{2}$, J.P. Martin ${ }^{3}$, S.F. Ashley, B.P. Crider ${ }^{4}$, M.T. McEllistrem ${ }^{4}$, E.E. Peters ${ }^{4}$, F.M. Prados-Estevez ${ }^{4}$, S.W. Yates ${ }^{4}$, and J.R. Vanhoy ${ }^{5}$

${ }^{1}$ Department of Physics, University of Guelph, Guelph, ON, N1G 2W1, Canada

${ }^{2}$ TRIUMF, Vancouver, BC, V6T 2A3, Canada

${ }^{3}$ Département de physique, Université de Montréal, Montreal QC, H3C 3J7, Canada

${ }^{4}$ Department of Physics and Astronomy, University of Kentucky, Lexington, KY 40506, USA

${ }^{5}$ Department of Physics, United States Naval Academy, Annapolis, MD 21402, USA

\begin{abstract}
A neutron tagging array based upon liquid deuterated scintillators is being developed for the study of neutron-rich systems. The DEuterated SCintillator Array for Neutron Tagging, or DESCANT, will serve as an auxiliary detector for both the TIGRESS and GRIFFIN $\gamma$-ray spectrometers located at TRIUMF's ISAC radioactive ion beam facility. DESCANT is comprised of 70 pseudohexaconical detectors of five varieties. The array is fully close-packed, subtends a downstream angle of $\theta=65^{\circ}$ and covers $92.6 \%$ of this solid angle or $1.08 \pi$ sr. Each detector is $150 \mathrm{~mm}$ thick and filled with Bicron BC-537 liquid deuterated scintillator. The white, red and blue detectors are viewed by $127 \mathrm{~mm}$ diameter Hamamatsu R4155 photomultiplier tubes while the yellow and green detectors are viewed by $78 \mathrm{~mm}$ diameter ET Enterprises $9822 \mathrm{~B}$ photomultiplier tubes. The aim of this work is to report on the mechanical design of DESCANT and the performance of a prototype detector measured using mono-energetic neutrons.
\end{abstract}

\section{Introduction}

The study of neutron-rich nuclei is at the forefront of nuclear structure research, and will continue to be for the next several decades [1]. For this purpose, large multi-detector $\gamma$-ray spectrometers coupled with auxiliary systems for neutron and light-charged particle detection have been shown to be highly efficient and highly selective when identifying reaction exit channels. As part of a program planned for study of neutron-rich nuclei at the TRIUMF-ISAC facility [2], the new neutron detector array DESCANT [3], capable of being coupled to both the TIGRESS [4, 5] and GRIFFIN [6] $\gamma$-ray spectrometers, was considered a necessity. In order to meet these needs, the DEuterated SCintillator Array for Neutron Tagging, DESCANT, was proposed and its construction is nearing completion. Coupled to a high-efficiency $\gamma$-ray spectrometer, DESCANT will allow a program of in-beam gammaray spectroscopy with neutron-rich beams. DESCANT, shown in Figure 1, is comprised of an array

\footnotetext{
ae-mail: jwong01@uoguelph.ca
} 

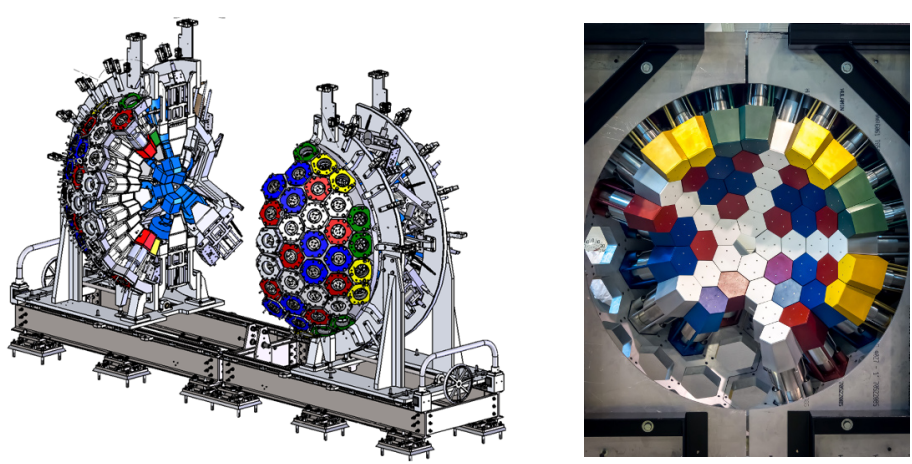

Figure 1. DESCANT coupled to GRIFFIN, a state-of-the-art high-efficiency $\gamma$-ray spectrometer (left). DESCANT shown partially assembled (right). The view is in the direction of the beam; DESCANT is attached on the downstream side of the $\gamma$-ray spectrometer.

of 70 detectors which provides a close-packed coverage of a spherical shell with an opening angle up to $65^{\circ}$.

\section{Detector Design}

The DESCANT array is comprised of 70 pseudohexaconical detector cells in five rings. The five basic shapes for the scintillator cans, referred to as white, red, blue, yellow and green, are shown in Figure 2. The full array consists of 20 white, 15 red, 15 blue, 10 yellow and 10 green detectors. The shapes of the individual detectors were designed to close-pack a semi-spherical shell to maximize the solid-angle coverage within a $65^{\circ}$ cone - the available opening angle of the forward hemisphere of the TIGRESS and GRIFFIN spectrometers. With close-packing, a solid angle of $1.08 \pi$ sr is achieved. The distance from the beam target located at the focal point of the neutron detector array to the front face of each can is $500 \mathrm{~mm}$.

The cells are $150 \mathrm{~mm}$ in height and are constructed with $1.5 \mathrm{~mm}$ thick Al, with a $9 \mathrm{~mm}$ thick quartz optical window. The front of the scintillator cans have a thickness of $2.7 \mathrm{~mm}$, and can be fitted with a removable $6.4 \mathrm{~mm}$ thick $\mathrm{Pb}$ shield. The white, red, and blue shapes are coupled to $127 \mathrm{~mm}$ diameter Hamamatsu R4155 photomultiplier tubes. The green and yellow shapes are coupled to $78 \mathrm{~mm}$ diameter ET Enterprises 9822B photomultiplier tubes. The anode signals will be sent via LMR400 low-loss cable directly into custom-built $1 \mathrm{GHz}$ 12-bit waveform digitizers where analysis of the pulse-shape will be performed. The dynode signals, which are unused, will be available via LMR200 cable for inspection and diagnostics.

\section{Liquid scintillator}

Liquid organic scintillators have long been used for fast-neutron detection, due to their fast response and the ability to use pulse-shape discrimination to distinguish between neutron and $\gamma$-ray interactions. DESCANT employs an innovative design that uses a deuterated-benzene liquid scintillator medium rather than the more traditional NE213 or its modern equivalent BC-501A. Due to the asymmetric nature of $n-d$ scattering in the center of mass, unlike n-p scattering that is isotropic, pulse-height spectra from the deuterated scintillator contain useful information on the initial neutron energy [7]. While deuterated detectors have been used in active-target experiments, and as $\gamma$-ray detectors in the 

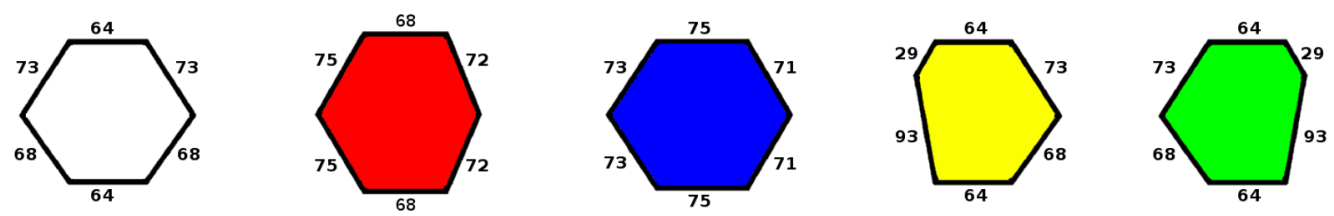

Figure 2. The five basic shapes of the DESCANT detectors designed to achieve close-packing. The dimensions in millimetres are given for the front face of each detector type.

presence of large thermal neutron fluxes, they had not been used in an array of detectors for fast neutrons.

A recent comparison between liquid deuterated scintillator, BC-537, produced by St. Gobain and the non-deuterated variety, NE213, [7] concluded that neutrons with energy as low as $60 \mathrm{keV}$ could be detected using BC-537. Furthermore, the relative efficiency between BC-537 and NE213 was approximately equal above $2 \mathrm{MeV}$. Below $2 \mathrm{MeV}$, the $\mathrm{n}$-d scattering cross section is lower than that of the corresponding n-p scattering, leading to a lower relative efficiency for BC-537. Finally, it was observed that the ability to discriminate between neutron and $\gamma$ events, is better for the deuterated scintillator than for the non-deuterated scintillator.

\section{DESCANT Detector Response}

The measurements described in the recent study cited above [7] were repeated using a large-volume white DESCANT detector. The preliminary analysis reveals that the use of a large volume of liquid deuterated scintillator does not greatly impact the quality of data that can be collected using a smaller quantity of scintillator.

The pulse-height spectra collected continue to display a definite peak structure, evident in the left panel of Figure 3. This peak structure will be combined with the time-of-flight to allow for a better determination of the initial neutron energy than with the time-of-flight alone.

The right panel of Figure 3 shows pulse-shape discrimination spectra collected using the zerocrossover-time method $[8,9]$. These spectra can be used to distinguish between neutron and $\gamma$-ray interactions. For each spectrum, the peak due to neutron interactions in the liquid scintillator is that occuring at the higher zero-crossing time. It is significant to note that the neutron- $\gamma$ discrimination capabilities demonstrated in the small volume of BC-537 [7] remain in the larger DESCANT detector.

The time resolution of the white detector was measured with a ${ }^{60} \mathrm{Co}$ calibration source using a fast plastic scintillator and yields a FWHM of $0.97 \mathrm{~ns}$.

\section{Summary}

A recent study established that the performance of the deuterated scintillator, BC-537, was comparable to and even exceeded that of the non-deuterated scintillator, NE213, as a neutron detector. It also clearly showed the existence of a peak-like structure in the pulse-height spectra. Similar measurements using a white DESCANT detector were conducted to demonstrate that the performance capabilities were retained by a large volume of deuterated scintillator.

The design of DESCANT presented in this work combined with the performance of the detectors shown from our preliminary tests indicate that DESCANT will be a very powerful experimental tool for nuclear structure physics. 

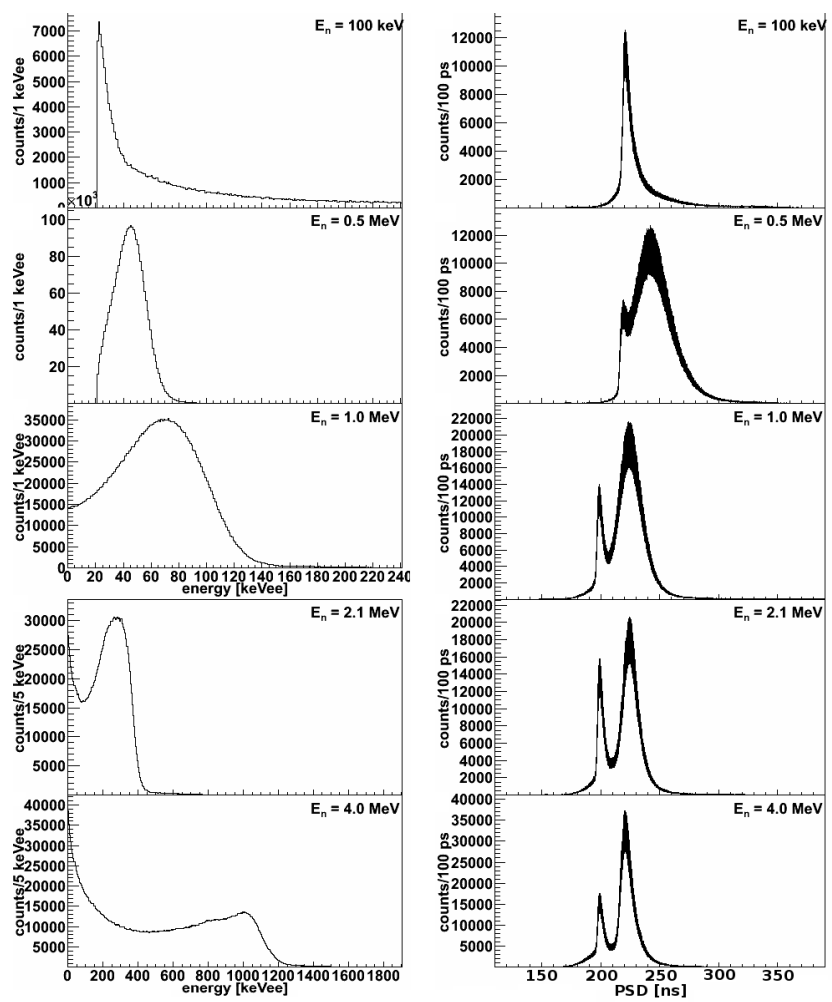

Figure 3. Pulse-height spectra (left) and pulse-shape discrimination spectra (right) measured using a white DESCANT detector for neutron energies from $100 \mathrm{keV}$ to $4.0 \mathrm{MeV}$.

\section{References}

[1] D.F. Geesamam, C.K. Gelbke, R.V.F. Janssens, B.M. Sherrill, Physics of a Rare Isotope Accelerator, Annual Review of Nuclear and Particle Science 56, 53-92 (2006).

[2] J. Dilling, R. Krücken, G.C. Ball, The ISAC Facility, Hyperfine Interactions, in press.

[3] P.E. Garrett, The DESCANT Spectrometer, Hyperfine Interactions, in press.

[4] C.E. Svensson, et. al., TIGRESS: TRIUMF-ISAC gamma-ray escape-suppressed spectrometer, Journal of Physics G: Nuclear and Particle Physics 31 (10) (2005).

[5] G. Hackman, C.E. Svensson, The TRIUMF-ISAC Gamma-Ray Escape-Suppressed Spectrometer, TIGRESS, Hyperfine Interactions, in press.

[6] C.E. Svensson, A. Garnsworthy, The GRIFFIN Spectrometer, Hyperfine Interactions, in press.

[7] V. Bildstein, et. al., Nuclear Instruments and Methods in Physics Research Section A: Accelerators, Spectrometers, Detectors and Associated Equipment 729, 188-197 (2013).

[8] T.K. Alexander, F.S. Goulding, An amplitude-insensitive system that distinguishes pulses of different shapes, Nuclear Instruments and Methods 13, 244-246 (1961).

[9] R.A. Winyard, J.E. Lutkin, G.W. McBeth, Pulse shape discrimination in inorganic and organic scintillators, I, Nuclear Instruments and Methods 95, 141-153 (1971). 